\title{
EXACT SOLUTION FOR STEADY PAINT FILM FLOW OF A PSEUDO PLASTIC FLUID DOWN A VERTICAL WALL BY GRAVITY
}

\author{
M.K.ALAM ${ }^{1 \dagger}$, M.T.RAHIM ${ }^{1}$, S.ISLAM $^{2 \dagger}$, AND A.M.SIDDIQUI $^{3 \dagger}$ \\ ${ }^{1}$ National University of Computer \& Emerging Sciences, Peshawar, Pakistan, \\ E-mail address: kamranalam2005@yahoo.com \\ ${ }^{2}$ Department of mathematics, Abdul Wali Khan University, Mardan, Pakistan, \\ E-mail address: saeed.sns@gmail.com \\ ${ }^{3}$ Pennsylvania State University, York Campus, 1031 Edgecombe aVenue, York, PA 17403 , \\ USA
}

\begin{abstract}
Here in this paper, the steady paint film flow on a vertical wall of a non-Newtonian pseudo plastic fluid for drainage problem has been investigated. The exact solution of the nonlinear problem is obtained for the velocity profile. Also the average velocity, volume flux, shear stress on the wall, force to hold the wall in position and normal stress difference have been derived. We retrieve Newtonian case, when material constant $\mu_{1}$ and relaxation time $\lambda_{1}$ equal zero. The results for co-rotational Maxwell fluid is also obtained by taking material constant $\mu_{1}=0$. The effect of the zero shear viscosity $\eta_{0}$, the material constant $\mu_{1}$, the relaxation time $\lambda_{1}$ and gravitational force on the velocity profile for drainage problem are discussed and plotted.
\end{abstract}

\section{INTRODUCTION}

Nowadays for different physical problems of Newtonian and non-Newtonian fluids an extensive literature is available and a lot of interest has been shown towards the study of exact, analytic and numerical solutions of these physical problems. The governing equations of nonNewtonian fluids are highly non-linear and it is also accepted now that in general, these equations are higher in order than the Navier-Stokes equations. Therefore, the exact solutions of these equations are very difficult and even for the case of viscous fluids. A lot of work has been carried out regarding the exact solutions for flows of non-Newtonian fluids $[1,2,3,4,5]$.

Over the past few decades there has been a growing recognition of the fact that many fluids of industrial significance do not obey the Newtonian postulate of linear relationship between the shear stress and shear rate. Therefore, these fluids are known as non-Newtonian fluids. Common examples of such fluids are slurries, pastes, gels, molten plastics and lubricants containing polymer additives. Various food stuffs such as honey and tomato sauce, the biological

Received by the editors March 30 2012; Revised August 24 2012; Accepted in revised form September 142012. 2010 Mathematics Subject Classification. 74F10.

Key words and phrases. Drainage problem; pseudo plastic fluid; Exact solution.

${ }^{\dagger}$ Corresponding authors. 
fluids like blood and synovial fluid naturally found in cavities of synovial joints also belong to the general class of the non-Newtonian fluids $[6,7]$. The simplest model of non-Newtonian fluids is the power law model and a special case of the power law equation, with $n<1$, is the pseudo plastic model. Commonly encountered non-Newtonian fluids like polymer solutions, paper pulps, detergents, oils and greases may be classified by the pseudo plastic fluid model [8]. The pseudo plastic fluids represent shear thinning fluids and, as far as we know, this class of non-Newtonian fluids received less attention in the literature.

Due to complexity of fluids there is not a single constitutive equation which can describe the properties of all non-Newtonian fluids. In view of this, several non- Newtonian fluid models have been proposed. There are many fluid models which describe the non-Newtonian behavior of the fluids but in recent years, the pseudo plastic fluid have attracted many researchers. Therefore, in the present analysis, we consider the steady, incompressible, parallel, laminar flow of a pseudo plastic fluid of paint flowing down on an infinite vertical wall. The thickness of the paint film is assumed to be $h$ and gravity acts in the negative $z$-direction (downward). The paint fall under the influence of gravity and there is no applied pressure driving the flow. Here, we also neglecting the change in the hydrostatic of (atmospheric pressure) the surrounding air. Therefore, our aim here, to find the effects of relaxation times on the steady flow of pseudo plastic paint ( fluid) flowing down on an infinite vertical wall.

Nowadays a lot of interest has been shown by researcher towards the study of the thin film flows of non-Newtonian fluids due to its wide spread applications in nonlinear sciences and engineering industries. The work on the thin film flows of non-Newtonian fluids under various configurations is relatively of recent origin. Siddiqui et al. [9] discussed the thin film flows of a third grade fluid down an inclined plane. In [10] they examined the thin film flows of PhanThein-Tanner (PTT) fluids on a moving belt. Siddiqui et al. [11] also considered the same flow of non-Newtonian fluids on a moving belt. The thin film flow of a fourth grade fluid down a vertical cylinder is also analyzed by Siddiqui et al. [12].

To the author's knowledge, no previous attempted has been reported to develop the governing equations for steady incompressible flow of a pseudo plastic fluid model in case of thin film paint flow. In this work it is intended to construct the equations for an incompressible pseudo plastic fluid model. The non-linear differential equations are solved for velocity field by means of exact method.

This paper is organized as follows: In section 2, the governing equations are developed. Section 3 deals with the formulation of the problem, flow equation, average velocity and volume flow rate. Result and discussion is given in section 4. The conclusion is given in section 5 .

\section{GOVERNING EQUATIONS}

The basic equations, governing the motion of an isothermal, homogeneous, incompressible fluid are:

$$
\begin{aligned}
\operatorname{div} \mathbf{V} & =0, \\
\rho \frac{D \mathbf{V}}{D t} & =\rho \mathbf{f}-\operatorname{grad} P+\operatorname{div} \mathbf{S},
\end{aligned}
$$


where $\rho$ is the constant fluid density, $\mathbf{V}$ is the velocity vector and $\mathbf{f}$ is the body force per unit mass, $P$ denotes the dynamics pressure, the $\frac{D}{D t}$ denotes the material derivative defined as

$$
\frac{D(*)}{D t}=\frac{\partial}{\partial t}(*)+(\mathbf{V} \cdot \nabla)(*) .
$$

$\mathbf{S}$ is the extra stress tensor which for pseudoplastic fluid model is defined as [13]:

$$
\mathbf{S}+\lambda_{1} \stackrel{\nabla}{\mathbf{S}}+\frac{1}{2}\left(\lambda_{1}-\mu_{1}\right)\left(\mathbf{A}_{1} \mathbf{S}+\mathbf{S} \mathbf{A}_{1}\right)=\eta_{0} \mathbf{A}_{1},
$$

where $\eta_{0}$ is the zero shear viscosity, $\lambda_{1}$ is the relaxation time and $\mu_{1}$ is the material constant. The first Rivlin-Ericksen tensor $\mathbf{A}_{\mathbf{1}}$ is defined as

$$
\mathbf{A}_{1}=(\operatorname{grad} \mathbf{V})+(\operatorname{grad} \mathbf{V})^{T} .
$$

The contravariant convected derivative denoted by super imposed $\nabla$ over $\mathbf{S}$, is defined as

$$
\stackrel{\nabla}{\mathbf{S}}=\frac{D \mathbf{S}}{D t}-\left\{(\operatorname{grad} \mathbf{V})^{T} \mathbf{S}+\mathbf{S}(\operatorname{grad} \mathbf{V})\right\}
$$

\section{FORMULATION OF THE PAINT FILM FLOW DOWN A VERTICAL WALL BY GRAVITY}

Consider steady, incompressible, parallel, laminar flow of paint flowing down on an infinite vertical wall. The thickness of the paint film is assumed to be $h$ and gravity acts in the negative $z$-direction (downward) which means that $g_{z}=-g$. The paint fall under the influence of gravity and there is no applied pressure driving the flow as shown in figure I. Neglecting the change in the hydrostatic of (atmospheric pressure) the surrounding air.

The boundary conditions for the problem are

(i) There is no-slip at the wall

$$
\text { at } \quad x=0, \quad w=0 .
$$

(ii) At the free surface, there is negligible shear

$$
\text { at } x=h, \quad S_{x z}=0 .
$$

where $S_{x z}$ is the shear stress component of pseudoplastic fluid.

We seek accordingly the velocity profile of the form

$$
\mathbf{V}=(0,0, w(x)),
$$

and extra stress tensor as

$$
\mathbf{S}=\mathbf{S}(x)
$$




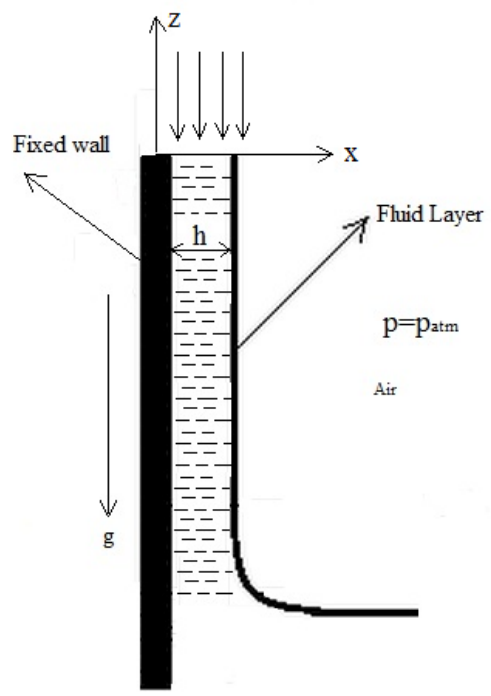

Fig I. A pseudo plastic film of paint falling by gravity along a vertical wall

By substituting (3.3) in (2.1) and (2.2), the continuity equation (2.1) is identically satisfied and the momentum equation (2.2) reduces to

$$
\begin{aligned}
& 0=\frac{d S_{x x}}{d x}+\rho f_{1}, \\
& 0=\frac{d S_{x z}}{d x}+\rho f_{3},
\end{aligned}
$$

where $f_{1}$ and $f_{3}$ are components of body force in $x$ and $z$ directions, respectively.

Since $z$ - axis is in upward direction and gravity acts in the negative $z$-direction (downward) which means that $g_{z}=-g$, so the above equations become

$$
\begin{aligned}
& 0=\frac{d S_{x x}}{d x} \\
& 0=\frac{d S_{x z}}{d x}-\rho g,
\end{aligned}
$$

since $S_{x x}$ is constant and integrating equation (3.8), we find that

$$
S_{x z}=\rho g x+C_{1},
$$

where $C_{1}$ is a constant of integration. Applying the free surface condition (3.2) on equation (3.9), we get

$$
S_{x z}=\rho g(x-h) .
$$


Using equation (2.4), (2.5), (3.3) and (3.4) in equation (2.3), we obtain the non-zero components of $\mathbf{S}$ as

$$
\begin{aligned}
& S_{x x}=-\left(\lambda_{1}-\mu_{1}\right)\left(\frac{d w}{d x}\right) S_{z x}, \\
& S_{z z}=\left(\lambda_{1}+\mu_{1}\right)\left(\frac{d w}{d x}\right) S_{z x}, \\
& S_{z x}=\frac{\eta_{0}\left(\frac{d w}{d x}\right)}{1+\left(\lambda_{1}^{2}-\mu_{1}^{2}\right)\left(\frac{d w}{d x}\right)^{2}} .
\end{aligned}
$$

On implementing one value of $S_{z x}$, we rewrite equation (3.10) as

or

$$
\frac{\eta_{0}\left(\frac{d w}{d x}\right)}{1+\left(\lambda_{1}^{2}-\mu_{1}^{2}\right)\left(\frac{d w}{d x}\right)^{2}}=\rho g(x-h) .
$$

$$
\left(\frac{\rho g}{\eta_{0}}\right)\left(\lambda_{1}^{2}-\mu_{1}^{2}\right)(h-x)\left(\frac{d w}{d x}\right)^{2}+\left(\frac{d w}{d x}\right)+\frac{\rho g}{\eta_{0}}(h-x)=0 .
$$

This is a first order non-linear differential equation which has to be solved with the help of no-slip condition (3.1), that is

$$
w=0, \text { at } x=0 .
$$

The above equation (3.15) is a quadratic equation in $\frac{d w}{d x}$, on solving this equation, we arrive at

$$
\frac{d w}{d x}=\frac{-1 \pm \sqrt{1-4\left(\frac{\rho g}{\eta_{0}}\right)^{2}\left(\lambda_{1}^{2}-\mu_{1}^{2}\right)(h-x)^{2}}}{2\left(\frac{\rho g}{\eta_{0}}\right)(h-x)\left(\lambda_{1}^{2}-\mu_{1}^{2}\right)} .
$$

On defining $m=\left(2 \frac{\rho g n}{\eta_{0}}\right)^{2}$, where $n^{2}=\left(\lambda_{1}^{2}-\mu_{1}^{2}\right)$ or $n \sqrt{m}=2 \frac{\rho g}{\eta_{0}} n^{2}$. Now considering the positive real part of equation (3.16), we have

$$
\frac{d w}{d x}=\frac{\sqrt{1-m(h-x)^{2}}-1}{n \sqrt{m}(h-x)} .
$$

On integrating equation (3.17), we get

$$
w(x)=\frac{1}{n \sqrt{m}} \int \frac{\sqrt{1-m(h-x)^{2}}-1}{(h-x)} d x .
$$


The solution of the above integration yields the general form as

$$
w(x)=\frac{1}{n \sqrt{m}}\left[\sqrt{1-m(h-x)^{2}}-\ln \left(\sqrt{1-m(h-x)^{2}}+1\right)\right]+C_{2} .
$$

where $C_{2}$ is a constant of integration and the above solution is the general form of equation (3.18). To find the integration constant $C_{2}$ we apply the boundary condition (3.1), the velocity profile $w(x)$ takes the form

$$
w(x)=\frac{1}{n \sqrt{m}}\left[\sqrt{1-m(h-x)^{2}}-\sqrt{1-m h^{2}}+\frac{\ln \left(\sqrt{1-m h^{2}}+1\right)}{\ln \left(\sqrt{1-m(h-x)^{2}}+1\right)}\right],
$$

substituting the values of $m$ and $n$, we arrive at the following form

$$
\begin{aligned}
w(x)= & \frac{\eta_{0}}{2 \rho g\left(\lambda_{1}^{2}-\mu_{1}^{2}\right)}\left[\sqrt{1-\frac{(2 \rho g)^{2}\left(\lambda_{1}^{2}-\mu_{1}^{2}\right)(h-x)^{2}}{\eta_{0}^{2}}}-\sqrt{1-\frac{(2 \rho g)^{2}\left(\lambda_{1}^{2}-\mu_{1}^{2}\right)}{\eta_{0}^{2}}} h^{2}\right. \\
& \left.+\frac{\ln \left(\sqrt{\left.1-\frac{(2 \rho g)^{2}\left(\lambda_{1}^{2}-\mu_{1}^{2}\right)}{\eta_{0}^{2}} h^{2}+1\right)}\right]}{\ln \left(\sqrt{1-\frac{(2 \rho g)^{2}\left(\lambda_{1}^{2}-\mu_{1}^{2}\right)}{\eta_{0}^{2}}(h-x)^{2}}+1\right)}\right]
\end{aligned}
$$

which is the velocity of the paint flow down on an infinite vertical wall. Here it should be noted that for $\lambda_{1}=\mu_{1}=0$, we get solution for Newtonian fluid. Also for $\mu_{1}=0$, the co-rotational Maxwell fluid $[6,7]$ recorded as shown below.

$$
w(x)=\frac{\eta_{0}}{2 \rho g \lambda_{1}^{2}}\left[\sqrt{1-\frac{(2 \rho g)^{2} \lambda_{1}^{2}(h-x)^{2}}{\eta_{0}^{2}}}-\sqrt{1-\frac{(2 \rho g)^{2} \lambda_{1}^{2}}{\eta_{0}^{2}} h^{2}}+\frac{\ln \left(\sqrt{1-\frac{(2 \rho g)^{2} \lambda_{1}^{2}}{\eta_{0}^{2}} h^{2}}+1\right)}{\ln \left(\sqrt{1-\frac{(2 \rho g)^{2} \lambda_{1}^{2}}{\eta_{0}^{2}}(h-x)^{2}}+1\right)}\right] .
$$

3.1. Normal Stress Difference. The expressions (3.11) to (3.13) show that the normal stress difference is given by

$$
S_{x x}-S_{y y}=-\frac{2 \lambda_{1} \eta_{0}\left(\frac{d v}{d x}\right)^{2}}{1+\left(\lambda_{1}^{2}-\mu_{1}^{2}\right)\left(\frac{d v}{d x}\right)^{2}}=-2 \lambda_{1}\left(\frac{d v}{d x}\right) S_{x y}
$$

which, with the help of (3.10) and (3.21), yields

$$
S_{x x}-S_{y y}=\frac{\lambda_{1} \eta_{0}}{\left(\lambda_{1}^{2}-\mu_{1}^{2}\right)}\left[1-\sqrt{1-\left(\frac{2 \rho g}{\eta_{0}}\right)^{2}\left(\lambda_{1}^{2}-\mu_{1}^{2}\right)(\delta-x)^{2}}\right] .
$$


The expression (3.23) reveals that the normal stress difference vanishes at the free surface $(x=\delta)$.

3.2. Force to Hold the wall in Position. The force $F$ per unit width to hold the wall in position can also be determined using the expression for shear stress at the belt surface. In fact, we have the formula

$$
\frac{F}{W}=\int_{0}^{H}\left(S_{x y}\right)_{x=0} d x
$$

where $H$ is the length of the belt. Using (3.10) and (3.24), we obtain

$$
\frac{F}{W}=-S_{t} H
$$

Equation (3.25) can also be used to determine the length $H$ of the belt, once the force per unit width is known.

3.3. Flow rate and average velocity of paint film flow. The flow rate per unit width is given by the formula

$$
Q=\int_{0}^{h} w(x) d x
$$

Substituting (3.21) in (3.26) and then integrating, we obtain the flow rate of pseudoplastic fluid as

$$
\begin{aligned}
Q & =h+\frac{(\sqrt{2}-1) \eta_{0}}{\sqrt{2 \rho g\left(\lambda_{1}^{2}-\mu_{1}^{2}\right)}} \operatorname{Arcsin}\left[\left(\sqrt{\frac{2 \rho g\left(\lambda_{1}^{2}-\mu_{1}^{2}\right)}{\eta_{0}}}\right) h\right]-\frac{3 h}{4} \sqrt{1-\frac{2 \rho g\left(\lambda_{1}^{2}-\mu_{1}^{2}\right) h^{2}}{\eta_{0}}} \\
& +(h-1) \log \left[1+\sqrt{1-\frac{2 \rho g\left(\lambda_{1}^{2}-\mu_{1}^{2}\right) h^{2}}{\eta_{0}}}\right]
\end{aligned}
$$

The average velocity $\bar{W}$ is given by

$$
\begin{aligned}
\bar{W} & =\frac{Q}{h}, \\
& =1+\frac{(\sqrt{2}-1) \eta_{0}}{h \sqrt{2 \rho g\left(\lambda_{1}^{2}-\mu_{1}^{2}\right)}} \operatorname{Arcsin}\left[\left(\sqrt{\frac{2 \rho g\left(\lambda_{1}^{2}-\mu_{1}^{2}\right)}{\eta_{0}}}\right) h\right]-\frac{3}{4} \sqrt{1-\frac{2 \rho g\left(\lambda_{1}^{2}-\mu_{1}^{2}\right) h^{2}}{\eta_{0}}} \\
& +\left(1-\frac{1}{h}\right) \log \left[1+\sqrt{1-\frac{2 \rho g\left(\lambda_{1}^{2}-\mu_{1}^{2}\right) h^{2}}{\eta_{0}}}\right] .
\end{aligned}
$$


3.4. Shear stress on the wall. The expression for the shear stress $\left.S_{x z}\right|_{x=0}$ on the wall is obtained by using the equation (3.13) as:

$$
\left.S_{x z}\right|_{x=0}=-\frac{h \eta_{0}^{2}\left(1+\sqrt{\frac{\eta_{0}+2 g h^{2} \rho\left(\mu_{1}^{2}-\lambda^{2}\right)}{\eta_{0}}}\right)}{2 g h^{2} \rho\left(\mu_{1}^{2}-\lambda^{2}\right)+\eta_{0}\left(2+h^{2} \lambda^{2}-h^{2} \mu_{1}^{2}+2 \sqrt{\frac{\eta_{0}+2 g h^{2} \rho\left(\mu_{1}^{2}-\lambda^{2}\right)}{\eta_{0}}}\right)^{(3.20)}}
$$

3.5. Vorticity. The vorticity function $\tilde{w}$ ( $z$-component of vorticity vector) of the flow is given by

$$
\tilde{w}=\frac{d w}{d x}=\frac{-1+\sqrt{1-\frac{4 \rho^{2} g^{2}}{\eta_{0}^{2}}(h-x)^{2}\left(\lambda_{1}^{2}-\mu_{1}^{2}\right)}}{2\left(\frac{\rho g}{\eta_{0}}\right)\left(\lambda_{1}^{2}-\mu_{1}^{2}\right)(h-x)} .
$$

We can recover the expression for vorticity of a Newtonian fluid upon taking the limits $\lambda_{1} \rightarrow 0$, $\mu_{1} \rightarrow 0$ of equation (3.30).

\section{RESULTS AND DISCUSSION}

Here, we consider steady, incompressible, parallel and laminar flow of paint flowing down on an infinite vertical wall. The thickness of the paint film is assumed to be $h$ and gravity acts in the negative $z$-direction (downward). The paint fall under the influence of gravity and there is no applied pressure driving the flow. Figures 2 to 6 show the behavior of the velocity profile for the pseudo plastic paint film fluid given in expression (3.21). The graphs in these figures are plotted to show the effect of variation of the non-Newtonian parameters on the velocity of the fluid. All these graphs are plotted by considering the parameters $\delta, U_{0}, g, \rho, \lambda_{1}, \mu_{1}$ and $\eta_{0}$. The pseudo plastic fluid velocity in expression (3.21) is valid only if the condition $0<\left(\frac{\rho g \delta}{\eta_{0}}\right)^{2}\left(\lambda_{1}^{2}-\mu_{1}^{2}\right)<\frac{1}{4}$ holds. Therefore, figures 3 and 4 show graphs that are plotted when $0<\left(\frac{\rho g \delta}{\eta_{0}}\right)^{2}\left(\lambda_{1}^{2}-\mu_{1}^{2}\right)<\frac{1}{4}$. Since the value of $\left(\frac{\rho g \delta}{\eta_{0}}\right)^{2}$ is always positive, the two cases are of $\lambda_{1}>\mu_{1}$ and $\lambda_{1}<\mu_{1}$. Figure 3 shows the effect of increasing the values of $\mu_{1}$ on the velocity profile (3.21) when $\lambda_{1}$ is fixed. It is obvious from the figure that the downward velocity decreases with increasing the value of $\mu_{1}$ and the fluid with lesser $\mu_{1}$ moves more freely under the action of gravity. But figure 4 reveals that when the value of $\mu_{1}$ is fixed and that of $\lambda_{1}$ is varied, the fluid with greater $\lambda_{1}$ moves downward more freely and increasingly as compared to the one with a higher value of $\mu_{1}$. In figure 5, the value of $\eta_{0}$ is varying and the effect of varying the value of $\eta_{0}$ on fluid velocity is observed by fixing other parameters. Figure 6 shows the behavior of velocity when the value of $h$ is increased and that of $\lambda_{1}, \mu_{1}$ and other parameters are kept fixed. 


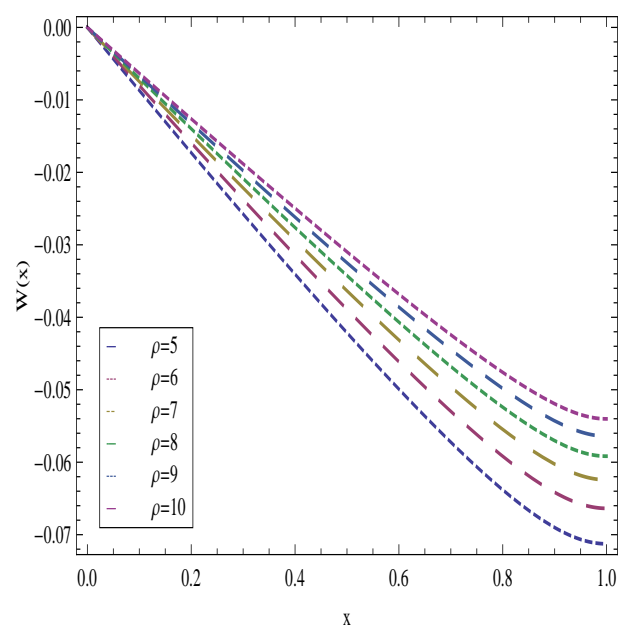

FIGURE 1 . The effect of constant density $\rho$ keeping $g=9 ; h=0.1 ; \eta_{0}=$ $0.2 ; \mu_{1}=0.6 ; \lambda_{1}=0.4$ on velocity profile for drainage problem.

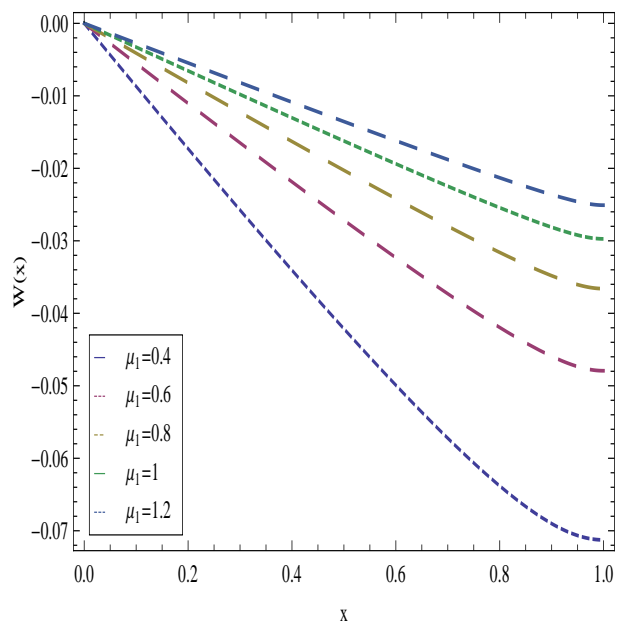

FIGURE 2. The effect of material constant $\mu_{1}$, keeping $\rho=5 ; g=9 ; h=$ $1 ; \eta_{0}=0.1 ; \lambda_{1}=2$ on velocity profile for drainage problem. 


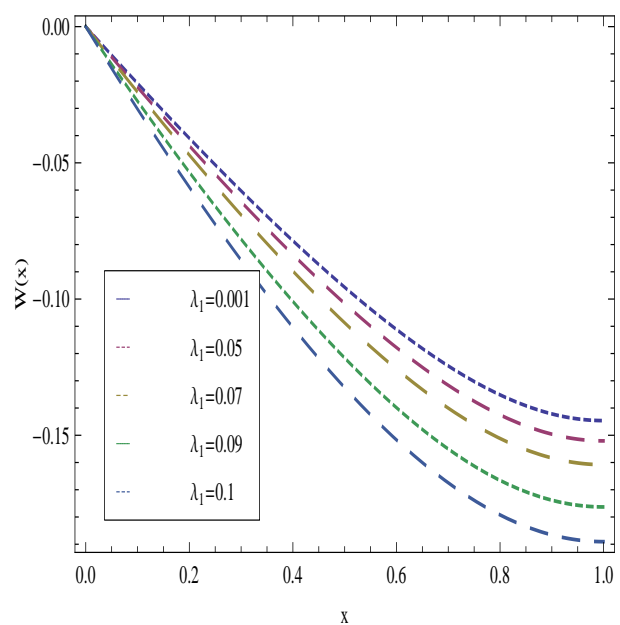

FIGURE 3. The effect on velocity profile of $\lambda_{1}$ keeping $\rho=5 ; g=9 ; h=$ $1 ; \eta_{0}=0.1 ; \mu_{1}=0.012$ for drainage problem.

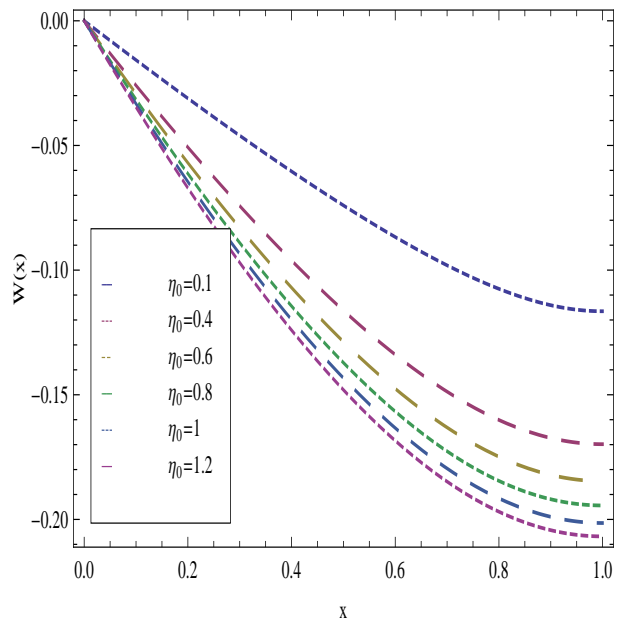

FIGURE 4. The effect on velocity profile of $\eta_{0}$ keeping $\rho=5 ; g=9 ; h=$ $1 ; \mu=0.2 ; \lambda_{1}=0.1$ for drainage problem. 


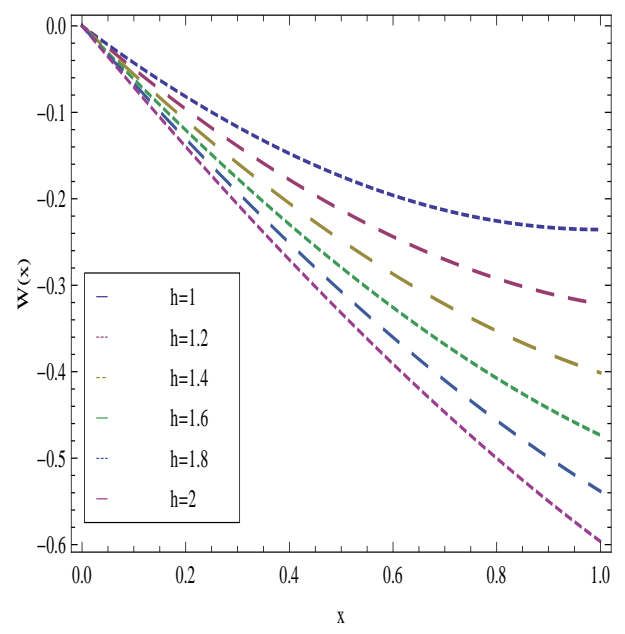

FIGURE 5 . The effect on velocity profile of $\eta_{0}$ keeping $\rho=5 ; g=9 ; h=$ $1 ; \mu=0.2 ; \lambda_{1}=0.1$ for drainage problem.

\section{CONCLUSION}

Thin film flow of pseudo plastic fluid down a vertically wall is analyzed. Exact analytical solution for fluid velocity is obtained. The condition under which this solution is valid is also stated. Expressions for some other useful quantities related to mechanics of the problem are derived. Graphs show the trend of the velocity profile with reference to the coordinate axes. Effects of non-Newtonian parameters on velocity profile are also presented through graphs.

\section{REFERENCES}

[1] Siddiqui, A.M., Islam, S. and Ghori, Q. K., Two-Dimensional Viscous Incompressible flows in a Porous Medium, J. of Porous Media, (2006), vol. 9(6), pp. 591-596.

[2] Islam, S. and Zhou, C.Y.(b), Exact Solutions of a Second Grade Fluid in a Porous Medium, Proc. NSC, Beijing, (2006).

[3] Islam, S. and Zhou, C.Y.(b), Certain inverse solutions of a second grade MHD aligned fluid flows in a porous medium, Journal of Porous Media, vol. 10(4), 401-408, (2007).

[4] Islam, S., Mohyuddin, M. R., and Zhou C. Y, Few exact solutions of non-Newtonian fluid in Porous Medium with Hall effects, Journal of porous Media, vol. 11(7), pp. 669-680,

[5] A. M. Siddiqui, M. KamranAlam, S. Islam, M. T. Rahim, Manzoor Elahi, A class of exact solutions of second grade fluid, Int. J. Phy. Sci. 6(24)5601-5608, 16 October, (2011).

[6] G. Astarita, G. Marrucci, Principle of non-Newtonian fluid mechanics, McGraw-Hill, London, (1974).

[7] R. B. Bird, R.C. Armstrong, O. Hassager, Dynamics of polymeric liquids, Fluid Mechanics, Wiley, New York, (1987).

[8] M. Moradi, Laminar flow heat transfer of a pseudoplastic fluid through a double pipe heat exchanger, Iranian Journal of Chemical Engineering, Vol. 3, No. 2, (2006); pp. 13-19.

[9] A.M. Siddiqui, R. Mahmood, Q.K. Ghori. Homotopy perturbation method for thin film flow of a third grade fluid down an inclined plane. Chaos, Solitons and Fractals 35 (2008) 140-147. 
[10] A.M. Siddiqui, R. Mahmood, Q.K. Ghori. Some exact solutions for the thin film flow of a PTT fluid. Physics Letters A 356 (2006) 353-356.

[11] A.M. Siddiqui, R. Mahmood, Q.K. Ghori. Thin film flow of non-Newtonian fluids on a moving belt. Chaos, Solitons and Fractals 33 (2007) 1006-1016.

[12] A. M. Siddiqui, R. Mahmood and Q. K. Ghori, Homotopy Perturbation Method for Thin Film Flow of a Fourth Grade Fluid down a Vertical Cylinder, Physical Letters A, Vol. 352, 2006, pp. 404-410. doi:10.1016/j.physleta.2005.12.033.

[13] J. A. Deiber, A. S. M. Santa Cruz, On non-Newtonian fluid flow through a tube of circular cross section, Lat. AM. J. Chem. Eng. Appl. Chem., Vol. 14, (1984); pp. 19-38. 